\title{
On the diffraction in the perturbative QCD
}

\author{
M.Braun \\ Department of High Energy physics, University of S.Petersburg, \\ 198904 S.Petersburg, Russia
}

April 17, 2018

\begin{abstract}
.
In the framework of the hard pomeron model it is shown that at large $Q^{2}$ the diffractive contribution to the structure functions rises as $Q^{0.3362}$ and much slowlier than the total contribution rising as $Q$.
\end{abstract}

SPbU-IP-1998-2 
1. The study of the four-gluon system in the high-colour limit $N \rightarrow \infty$ reveals that the diffractive amplitude turns out to be totally given by a contribution from the triple pomeron interaction [1], in agreement with the colour dipole picture [2]. In the latter approach the triple pomeron amplitude has recently been extensively studied in the high diffractive mass $M$ region, in relation to the diffractive structure functions [3]. With the c.m. energy squared $s, s_{1}=M^{2}$ and $s / s_{1}$ all being asymptotically large, the amplitude factorizes and, apart from logarithmic factors, becomes similar to the old triple pomeron contribution in the ReggeGribov picture with a triple pomeron coupling

$$
\gamma_{3 P}(\kappa)=\frac{32 \alpha_{s}^{2} N}{\kappa} B
$$

where $\kappa$ is the momentum transferred to the target and $B$ is a known number [4]

$$
B=\frac{g_{3 P}}{(2 \pi)^{4}}=\frac{7766.679 \ldots}{(2 \pi)^{4}} \simeq 4.983 \ldots
$$

However it is clear that the total contribution from this asymptotic region is neglegible at high $s$, the bulk of diffraction coming from the region of lower $M, \alpha_{s} \ln M \sim 1$, where the initial pomeron coupled to the projectile is not in its asymptotic regime. Because of that the total diffractive contribution (that is, integrated over $M$ ) looks much more complicated and even not factorizable in general.

In this note we want to demonstrate that in the particular case of a highly virtual photon projectile, relevant for the structure functions with $Q^{2} \rightarrow \infty$, the total diffractive contribution nevertheless factorizes and has a well-defined behaviour with $Q^{2}$ as $Q^{0.3662}$. So it rises with $Q^{2}$ much slowlier than the non-diffractive contribution given by a single pomeron exhange, which behaves as $Q$. This fact may serve as an additional signature of the hard pomeron in the diffractive events.

2. The diffractive amplitude (or rather its discontinuity in $s_{1}$ ) corresponding to the triple pomeron interaction is given by

$$
\begin{gathered}
D=-\frac{\alpha_{s}^{5}}{\pi^{3}} N^{2}\left(N^{2}-1\right) \frac{s^{2}}{s_{1}} \int \prod_{i=1}^{3} d^{2} r_{i} \frac{r_{1}^{2} \nabla_{1}^{4}}{r_{2}^{2} r_{3}^{2}} \\
\exp \left(-i \kappa\left(r_{2}+r_{3}\right) / 2\right) \phi_{1}\left(s_{1}, 0, r_{1}\right) \phi_{2}\left(s_{2}, \kappa, r_{2}\right) \phi_{2}\left(s_{2},-\kappa,-r_{3}\right) \delta^{2}\left(r_{1}+r_{2}+r_{3}\right)
\end{gathered}
$$

Here $\phi_{i}(s, \kappa, r)$, describe the pomerons coupled to the projectile $(i=1)$ and target $(i=2)$, with the energetic variable $s$, momentum $\kappa$ and the intergluon transverse distance $r ; s_{2}=$ $s / s_{1}$. The pomerons are joined by a triple pomeron vertex, whose form was obtained in [4] in the high-colour limit. We shall be interested in the total diffractive contribution integrated over the diffractive mass. In our normalization

$$
\frac{d \sigma^{D}}{d \kappa^{2}}=\int d s_{1} \frac{D}{16 \pi^{2} s^{2}}
$$

The pomerons $\phi_{1(2)}$ can be obtained by using the Green function of the BFKL equation for a given total momentum $G_{l}\left(s, r, r^{\prime}\right)$. For the projectile (see [5]):

$$
\phi_{1}(s, \kappa, r)=\int d^{2} r^{\prime}\left(G_{s}(\kappa, r, 0)-G_{s}\left(\kappa, r, r^{\prime}\right) \rho_{1}\left(r^{\prime}\right)\right.
$$

Here $\rho_{1}(r)$ is the colour density of the projectile as a function of the intergluon distance with the colour factor $(1 / 2) \delta_{a b}$ and $g^{2}$ separated. As mentioned, for the projectile we choose a 
highly virtual photon with $p_{1}^{2}=-Q^{2} \leq 0$, which splits into $q \bar{q}$ pairs of different flavours. For the transverse photon [5]

$$
\rho_{1}^{(T)}(r)=\frac{e^{2}}{4 \pi^{3}} \sum_{f=1}^{N_{f}} Z_{f}^{2} \int_{0}^{1} d \alpha\left(m_{f}^{2} \mathrm{~K}_{0}^{2}\left(\epsilon_{f} r\right)+\left(\alpha^{2}+(1-\alpha)^{2}\right) \epsilon_{f}^{2} \mathrm{~K}_{1}^{2}\left(\epsilon_{f} r\right)\right)
$$

where $\epsilon_{f}^{2}=Q^{2} \alpha(1-\alpha)+m_{f}^{2}$ and $m_{f}$ and $Z_{f}$ are the mass and charge of the quark of flavour $f$. For the longitudinal photon

$$
\rho_{1}^{(L)}(r)=\frac{e^{2}}{\pi^{3}} Q^{2} \sum_{f=1}^{N_{f}} Z_{f}^{2} \int_{0}^{1} d \alpha \alpha^{2}(1-\alpha)^{2} \mathrm{~K}_{0}^{2}\left(\epsilon_{f} r\right)
$$

For the hadronic target, we assume an expression similar to (4) with a colour density $\rho_{2}(r)$ non-perturbative and its explicit form unknown. I

Presenting the $\delta$-function in (2) as an integral over the auxiliary momentum $q$ we rewrite (2) in the form

$$
D=\frac{\alpha_{s}^{5}}{\pi^{3}} N^{2}\left(N^{2}-1\right) \frac{s^{2}}{s_{1}} \int \frac{d^{2} q}{(2 \pi)^{2}} \chi_{1}\left(s_{1}, 0, q+\kappa / 2\right) \chi_{2}^{2}\left(s_{2}, \kappa, q\right)
$$

where

$$
\chi_{1}(s, 0, q)=\int d^{2} r r^{2} \nabla^{4} \phi_{1}(s, 0, r) \exp i q r
$$

and

$$
\chi_{2}(s, \kappa, q)=\int d^{2} r r^{-2} \phi_{2}(s, \kappa, r) \exp i q r
$$

The two pomerons coupled to the target are in their asymptotic regime. So $\chi_{2}$ can be found using an asymptotic expression for the pomeron Green function, in the same way as in [6], to which paper we refer for the details. One obtains then

$$
\chi_{2}(s, \kappa, q)=8 s^{\Delta}(\pi / \beta \ln s)^{3 / 2} f(\kappa) J(\kappa, q)
$$

where $\Delta=\left(\alpha_{s} N / \pi\right) 4 \ln 2$ is the pomeron intercept, $\beta=\left(\alpha_{s} N / \pi\right) 14 \zeta(3)$,

$$
J(\kappa, q)=\int \frac{d^{2} p}{2 \pi} \frac{1}{|\kappa / 2+p||\kappa / 2-p||q+p|}
$$

and $f(\kappa)$ desribes the coupling of the lower pomeron to the target

$$
\int d^{2} R d^{2} r \frac{\exp (i \kappa R) r \rho_{2}(r)}{|R+r / 2||R-r / 2|}=\pi f(\kappa)
$$

At small $\kappa F_{2}$ diverges logarithmically:

$$
f \simeq-4(R / N) \ln \kappa, \quad \kappa \rightarrow 0
$$

where

$$
R=\frac{N}{2} \int d^{2} r r \rho_{2}(r)
$$

\footnotetext{
${ }^{1}$ If we put (4) into (2) and use explicit expressions for the Green functions $G$, we obtain the diffractive amplitude $D$ in the form given in [3] with an additional factor $1 / 16$. A part of it, 1/8, is due to our rule to include a factor 2 into the densities $\rho$. The rest factor $1 / 2$, in our opinion, comes from using in [3] the double dipole density without a symmetrizing one half.
} 
has a meaning of the average target dimension.

3. For the pomeron coupled to the projectile we have to use the exact Green function. Due to azimuthal symmetry we can retain only terms with zero orbital momentum in it:

$$
G_{0}\left(s, r, r^{\prime}\right)=(1 / 8) r r^{\prime} \int_{-\infty}^{\infty} \frac{d \nu s^{\omega(\nu)}}{\left(\nu^{2}+1 / 4\right)^{2}}\left(r / r^{\prime}\right)^{-2 i \nu}
$$

where

$$
\omega(\nu)=2\left(\alpha_{s} N / 2 \pi\right)(\psi(1)-\operatorname{Re} \psi(1 / 2+i \nu))
$$

If we take this expression, put it into $\chi_{1}$, Eq. (8) and integrate first over $s_{1}$, as indicated in (3), and afterwards over $r$, we obtain

$$
\int d s_{1} s_{1}^{-1-2 \Delta} \chi\left(s_{1}, 0, q\right)=-\frac{4 \pi}{q} \int \frac{d^{2} r^{\prime}}{(2 \pi)^{2}} r^{\prime} \rho_{1}\left(r^{\prime}\right) I\left(q, r^{\prime}\right)
$$

where $I\left(q, r^{\prime}\right)$ is the remaining integral over $\nu$ :

$$
I\left(q, r^{\prime}\right)=\int d \nu \frac{\left(q r^{\prime} / 2\right)^{2 i \nu}}{2 \Delta-\omega(\nu)} \frac{\Gamma(1 / 2-i \nu)}{\Gamma(1 / 2+i \nu}
$$

The integral (18) can be calculated as a sum of residues of the integrand at points $\nu=$ $\pm i x_{k}, 0<x_{1}<x_{2}<\ldots$, at which

$$
2 \Delta-\omega(\nu)=0
$$

Residues in the upper semiplane are to be taken if $q r_{1} / 2>1$ and those in the lower semiplane if $q r_{1} / 2<1$. Thus we obtain

$$
I\left(q, r_{1}\right)=\frac{2 \pi^{2}}{\alpha_{s} N} \sum_{k} c_{k}^{( \pm)}\left(q r_{1} / 2\right)^{ \pm 2 x_{k}}
$$

where

$$
c_{k}^{( \pm)}=\frac{\Gamma\left(1 / 2 \mp x_{k}\right) / \Gamma\left(1 / 2 \pm x_{k}\right)}{\psi^{\prime}\left(1 / 2-x_{k}\right)-\psi^{\prime}\left(1 / 2+x_{k}\right)}
$$

and the signs should be chosen to always have $\left(q r_{1} / 2\right)^{ \pm 2 x_{k}}<1$.

The first three roots of Eq. (19) are

$$
x_{1}=0.3169, \quad x_{2}=1.3718, \quad x_{3}=2.3867
$$

with the corresponding coefficients $c_{k}^{( \pm)}$

$$
\begin{gathered}
c_{1}^{(+)}=0.1522, \quad c_{2}^{(+)}=-0.1407, \quad c_{3}^{(+)}=0.03433, \\
c_{1}^{(-)}=0.007866, \quad c_{2}^{(-)}=-0.001802, \quad c_{3}^{(-)}=0.004494,
\end{gathered}
$$

Returning to Eq. (3) for $d \sigma / d \kappa^{2}$ as an integral of the amplitude $D$ and putting expressions for $\chi_{2}$ and the integrated $\chi_{2}$, Eqs. (10) and (17), into the latter we obtain

$$
\begin{gathered}
\frac{d \sigma^{D}}{d \kappa^{2}}=32 \pi \alpha_{s}^{4} N\left(N^{2}-1\right) \frac{s^{2 \Delta}}{(\beta \ln s)^{3}} f^{2}(\kappa) \sum_{k} \int d^{2} r r \rho_{1}(r) \\
{\left[c_{k}^{(+)}\left(\frac{r}{2}\right)^{2 x_{k}} B_{k}^{(+)}(r, \kappa)+c_{k}^{(-)}\left(\frac{r}{2}\right)^{-2 x_{k}} B_{k}^{(-)}(r, \kappa)\right]}
\end{gathered}
$$


where

$$
B_{k}^{( \pm)}(r, \kappa)=\int \frac{d^{2} q}{(2 \pi)^{2} q} q^{ \pm 2 x_{k}} J^{2}(\kappa, q-\kappa / 2) \theta( \pm(2 / r-q))
$$

As we observe, in the general case the factorization property is lost: the integrals $B^{( \pm)}$ depend nontrivially both on the projectile and target variables. However one can see that this property is restored in the limit of high $Q^{2}$, relevant for the hadronic structure functions. In fact, in this limit the characteristic values of $r$ are small: $r \sim 1 / Q$. Let us study how the integrals $B^{( \pm)}$behave at small $r$. In $B^{( \pm)}$evidently large values of $q$ are essential. The integrals $J$ behave as $\ln q / q$ at $q \rightarrow \infty$. This leads to the following behaviour.

$$
\begin{gathered}
B_{k}^{(+)}(r, \kappa) \sim r^{1-2 x_{k}} \text { if } 2 x_{k}>1 \text { and } \sim \text { const if } 2 x_{k}<1 \\
B_{k}^{(-)}(r, \kappa) \sim r^{1+2 x_{k}}
\end{gathered}
$$

Combining this with other factors depending on $r$ we see that all terms multiplying $\rho_{1}$ in the integrand behave as $r^{2}$ at small $r$, except the first term with $k=1$, which, due to $2 x_{1}<1$, behaves as $r^{1+2 x_{1}}$. Evidently this term gives the dominant contribution in the limit $Q^{2} \rightarrow \infty$, when (24) simplifies to

$$
\frac{d \sigma^{D}}{d \kappa^{2}}=2^{5-2 x_{1}} \pi e^{2} Z^{2} \alpha_{s}^{4} N\left(N^{2}-1\right) c_{1}^{(+)} \frac{s^{2 \Delta}}{(\beta \ln s)^{3}} f^{2}(\kappa) \frac{b_{1} B_{1}}{Q^{1+2 x_{1}} \kappa^{1-2 x_{1}}}
$$

where $Z^{2}=\sum_{f} Z_{f}^{2}$,

$$
e^{2} Z^{2} b_{1}=Q^{1+2 x_{1}} \int d^{2} r r^{1+2 x_{1}} \rho_{1}(r)
$$

(it does not depend on $Q$ ) and

$$
B_{1}=\kappa^{1-2 x_{1}} \int \frac{d^{2} q}{(2 \pi)^{2} q} q^{2 x_{1}} J^{2}(\kappa, q-\kappa / 2)
$$

(it does not depend on $\kappa)$. In $(28) J(\kappa, q)$ is given by (11). With $x_{1}=0$ (28) gives the number $B$, Eq. (1). Numerical calculations give

$$
b_{1}^{(T)}=0.3145, b^{(L)}=0.04377, B_{1}=17.93
$$

So the anomalous dimension in (28) raises the effective triple pomeron coupling more than three times. Indeces $T, L$ refer to the transverse or longitudinal photon projectile.

As a rtesult, we observe that in the high- $Q$ limit the expression for $d \sigma^{D} / d \kappa^{2}$ fully factorizes in the projectile and target. Its dependence on $Q$ and $\kappa$ turns out to be intermediate between the eikonal and asymptotic triple pomeron predictions. It vanishes at large $Q$ as $1 / Q^{1+2 x_{1}}$, faster than the single pomeron exchange and asymptotic triple pomeron $(\sim 1 / Q)$ but not so fast as the eikonal prediction $1 / Q^{2}$. It is also singular at $\kappa \rightarrow 0$, but the singularity is weaker than predicted by the asymptotic triple pomeron.

4. The diffractive contributions to the structure functions are obtained from (26) in the standard manner:

$$
F_{2}^{D}\left(x, Q^{2}, \kappa\right)=\frac{Q^{2}}{\pi e^{2}} \frac{d \sigma^{D}}{d \kappa^{2}}, s \rightarrow 1 / x
$$

(one has to sum the contributions from the transverse and longitudinal photon projectiles). From (30) one concludes that at large $Q^{2}$ the diffractive contribution behaves as $Q^{1-2 x_{1}}=$ 
$Q^{0.3362}$. This is to be compared with the non-diffractive contribution given by a single pomeron exchange in the same approach:

$$
F_{2}\left(x, Q^{2}\right)=\frac{11}{32} Z^{2} \alpha_{s}^{2} \frac{N^{2}-1}{N} \sqrt{\frac{\pi}{\beta \ln s}} s^{\Delta} Q R \exp \left(-\frac{\ln ^{2} Q R}{\beta \ln s}\right)
$$

At $\ln ^{2} Q R<<\beta \ln s$ it roughly behaves as $Q$ and so dominates over the diffractive contribution at a given $x$ and rising $Q$. However it rises with $1 / x$ only as $x^{-\Delta}$ whereas the diffractive contribution rises as its square (modulo logarithms). So in different regions of the $x, Q^{2}$ space the relative magnitude of the diffractive and non-diffractive contributions may be different.

To have an idea about the numerical magnitude of the diffractive contribution we have to make some specific assumptions about the hadronic colour density $\rho_{2}(r)$ which enters the form -factor $f(\kappa)$, Eq.(12). In accordance with (14) it shoud be normalized at $\kappa=0$ as

$$
(N / 2) \int d^{2} r \rho_{2}(r)=1, \quad \kappa=0
$$

However its form as a function of $r$ and $\kappa$ is unknown. To avoid introduction of several hadronic scales and also guided by the form of the photonic density $\rho_{1}(r)$ at large $Q^{2}$ and arbitrary $\kappa[1]$, we choose

$$
\rho_{2}(r)=\frac{\alpha^{2}}{\pi N} e^{-r(\alpha+\kappa)}
$$

The single dimensional parameter $\alpha$ is related to the average target dimension as $\alpha=2 / R$. With this choice we obtain

$$
f(\kappa)=(R / N) \tilde{f}(\kappa / \alpha)
$$

where

$$
\tilde{f}(\kappa)=\int \frac{d^{2} p}{\pi p|p+\kappa|} \frac{2(1+|\kappa|)^{2}-(p+\kappa / 2)^{2}}{\left((1+|\kappa|)^{2}+(p+\kappa / 2)^{2}\right)^{5 / 2}}
$$

With our choice the form factor $f$ goes down with $\kappa$ as a power. At $\kappa \rightarrow 0$ it blows up logarithmically (see Eq. (13)).

Integrating (30) over all $\kappa$ we get the total diffractive contribution to the structure function corresponding to transitions into the proton intermediate states

$$
F_{2}^{D}\left(x, Q^{2}\right)=64 Z^{2} \alpha_{s}^{4} \frac{N^{2}-1}{N} b_{1} B_{1} c(Q R)^{1-2 x_{1}} \frac{s^{2 \Delta}}{(\beta \ln s)^{3}}
$$

where the number $a$ is given by

$$
a=\int_{0}^{\infty} d \kappa \kappa^{2 x_{1}} \tilde{f}^{2}(\kappa) \simeq 6.033
$$

The ratio of the diffractive to non-diffractive contributions results

$$
F_{2}^{D} / F_{2}=c \alpha_{s}^{2}(Q R)^{-2 x_{1}} \frac{s^{\Delta}}{(\beta \ln s)^{5 / 2}} \exp \left(\frac{\ln ^{2} Q R}{\beta \ln s}\right), s \rightarrow 1 / x
$$

with a coefficient $c$ given by

$$
c=\frac{2048}{11 \sqrt{\pi}} a c_{1}^{(+)} B_{1}\left(b_{1}^{(T)}+b_{1}^{(L)}\right)
$$

In Table we present values of this ratio at $x=10^{-4}, 10^{-5}, 10^{-6}$ and $Q^{2}=10,100,1000(\mathrm{GeV} / \mathrm{c})^{2}$. We have chosen $\Delta=0.282$ and $1 / R=0.522 \mathrm{GeV}$ (see [3], our $R$ is their $r_{0} / 2$ ). As one observes the $x$-dependence of the ratio is quite weak in this region. On the contrary the $Q^{2}$ 
dependence is significant: the relative contribution of the diffractive events goes down by a factor of two as $Q^{2}$ rises from 10 to $1000(\mathrm{GeV} / \mathrm{c})^{2}$. The total relative number of diffractive events reaches $\sim 10 \%$ at the smallest values of $x$ and $Q^{2}$ in the studied interval.

\section{References}

1. M.A.Braun and G.P.Vacca, Bologna univ. preprint, hep-ph/9711486.

2. A.Mueller, Nucl. Phys.,B415 (1994) 373.

R.Peschanski, Phys. Lett. B409 (1997) 49.

3. A.Bialas, H.Navelet and R.Peschanski, Saclay preprints hep-ph/9711236, hep-ph/9711442.

4. G.P.Korchemsky, preprint LPTHE-Orsay-97-62; hep-ph/9711277.

5. N.N.Nikolaev and B.G.Zakharov, Z. Phys. C49 (1991) 607.

6. M.A.Braun, Z.Phys. C71 (1996) 123. 


\section{Table}

Ratio of the diffractive to nondiffractive contributons to $F_{2}\left(x, Q^{2}\right)$

\begin{tabular}{|r|c|c|c|}
\hline$Q^{2}(\mathrm{GeV} / \mathrm{c})^{2}$ & $x=10^{-4}$ & $x=10^{-5}$ & $x=10^{-6}$ \\
\hline 10 & 0.0750 & 0.0789 & 0.0932 \\
\hline 100 & 0.0512 & 0.0502 & 0.0566 \\
\hline 1000 & 0.0413 & 0.0366 & 0.0385 \\
\hline
\end{tabular}

